\title{
Search for Leptoquarks and Lepton Flavor Violation at HERA
}

\author{
Stefan Schmitt* \\ DESY, Germany \\ E-mail: Sschmitt@mail.desy.de
}

\begin{abstract}
The data collected with the $e p$ collider HERA at DESY in the years 1994-2000 at a centre-ofmass energy of $300-320 \mathrm{GeV}$ has been searched for Leptoquarks and lepton-flavour violation. Leptoquarks are searched in the decay channels $L Q-\rightarrow \ell q(\ell=e, v, \mu, \tau)$. For masses below the centre-of-mass energy a leptoquark signals would show up as a resonance in the $\ell q$ massspectra. At higher masses the signal may show up as a four-fermion interaction. No sign of processes involving leptoquarks has been found and limits on the leptoquark masses are set as a function of the leptoquark mass. For models involving lepton-flavour violation limits on the contact interaction term $\frac{\lambda_{\text {eq }} \lambda_{\mu, \tau q_{\beta}}}{M^{2}}$ are given.
\end{abstract}

International Europhysics Conference on High Energy Physics

July 21st - 27th 2005

Lisboa, Portugal

${ }^{*}$ Speaker. 


\section{Introduction}

Leptoquarks are bosonic objects which carry both leptonic and baryonic quantum numbers. They may couple directly to a lepton-quark pair. The couplings of such vertices are denoted $\lambda_{\ell q}$, where $\ell$ and $q$ denote the lepton and quark flavour, respectively. A new quantum number $F$ is introduced, where $F=3 \times B+L$ is the sum of three times the baryon number and the lepton number.

At the $e p$ collider HERA, a leptoquark coupling to an electron or positron and a quark may be produced by the fusion of the incoming $e^{+}$or $e^{-}$and a quark from the incoming proton. If the leptoquark mass is below the centre-of-mass energy of HERA $(320 \mathrm{GeV})$ resonant production in the $s$ channel is dominant. At higher masses the $u$ channel may contribute significantly. If only first generation leptons are present in both the production and the decay, the process may interfere with standard model processes (deep inelastic scattering). On the other hand, if a $\mu$ of $\tau$ lepton are present in the final state, the process violates lepton-flavour.

\section{Searches for leptoquarks coupling to 1st generation leptons}

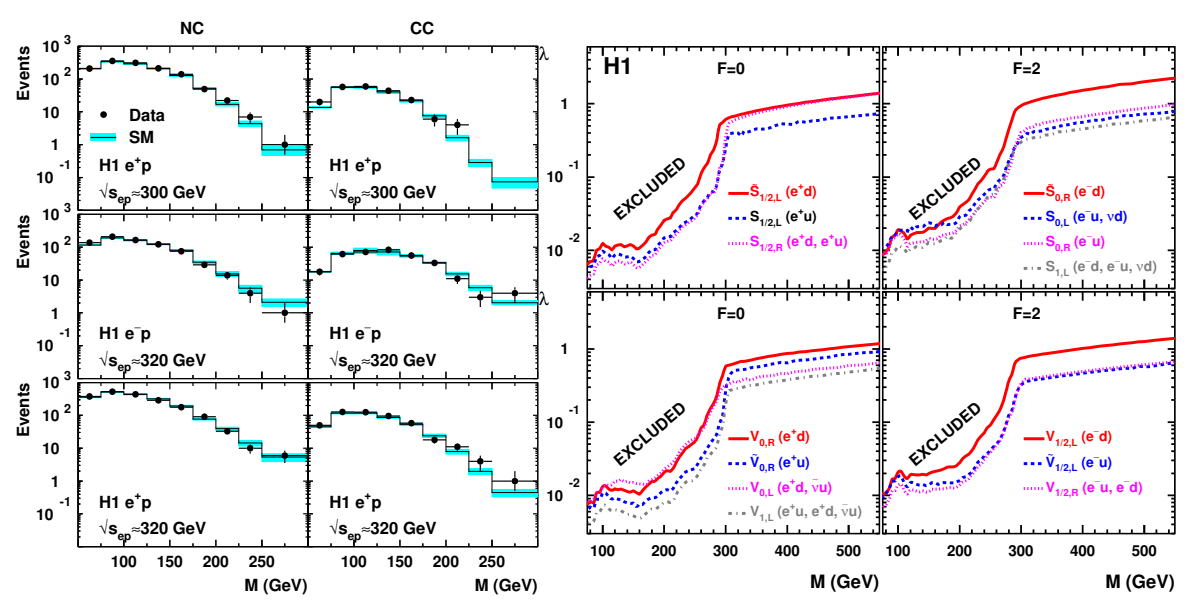

Figure 1: Electron quark and neutrino quark mass spectra, leptoquark limits

Leptoquarks coupling to $e q$ or $v q$ have been searched in the data collected by the H1 and ZEUS experiments at HERA in the years 1994-2000. Both experiments have analysed datasets corresponding to an integrated luminosity of about $100 \mathrm{pb}^{-1}$ in $e^{+} p$ collisions and $15 \mathrm{pb}^{-1}$ in $e^{-} p$ collisions. The $e^{+} p$ and $e^{-p}$ datasets are largely complementary when looking for $F=0$ and $F=2$ type leptoquarks. Leptoquark searches are performed by looking for deviations of the reconstructed leptoquark-mass and decay angle spectra from the standard-model expectation (deep inelastic scattering). No evidence for such deviations has been found, and mass-dependent exclusion limits on $\lambda_{e q}$ are set. Such limits have been calculated using the generic leptoquark models described by Buchmüller, Rückl and Wyler [1].

Mass spectra and limits obtained by the $\mathrm{H} 1$ collaboration are shown in Fig. 1. The limits are shown on the 14 types of leptoquarks which are accessible in $e p$ reactions. For masses below 
$300 \mathrm{GeV}$ the limits on $\lambda$ are from resonant leptoquark production, whereas at high masses contact interactions take over and the limits is valid for constant $\lambda / \mathrm{m}$. At low masses the exclusion limits on $\lambda$ are better than $10^{-2}$ for some cases.

In the Buchmüller, Rückl, Wyler model the branching ratio of the leptoquarks to $e q$ and $v q^{\prime}$ are fixed. More generic models are studied in Fig. 2, where this branching ratio $\beta_{e}$ is variable. For

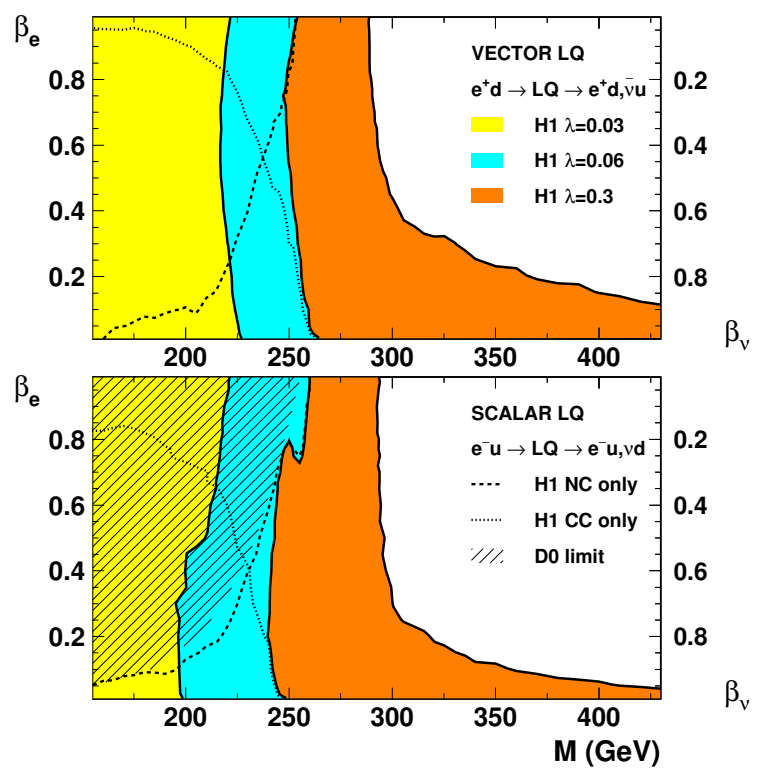

Figure 2: Leptoquark limits with variable branching ratio.

a coupling $\lambda_{e}=0.3$ one can see a smooth transition to the contact interaction regime at low $\beta_{e}$ and high masses. More details about the HERA data analyses may be found in ref [2, 4].

\section{Searches for lepton-flavour violation}

The H1 and ZEUS data has been searched for lepton-flavour violation mediated by leptoquarks. Such processes have been probed by searching for isolated $\mu$ or $\tau$ leptons and a jet. Corresponding lepton-quark mass-spectra are shown in figure 3. No evidence for an excess production of such events has been found, and limits on lepton-flavour violation leptoquark models are set.

In figure 4a limits on resonant leptoquark production and successive decay in the reaction $e p \rightarrow \ell X(\ell=\mu, \tau)$ are shown. Production and decay are decoupled, and limits are given both in terms of $\lambda_{e q_{1}} \times \sqrt{\beta_{\ell q}}$ and for the conventional assumption $\lambda_{e q_{1}}=\lambda_{\ell q}$. At high masses the interaction is a four fermion interaction. Limits on the relevant parameter $\frac{\lambda_{e q \alpha} \lambda_{\mu, \tau q_{\beta}}}{M^{2}}$ are shown in Figure $4 \mathrm{~b}$, together with indirect limits from low energy experiments.

More details about searches for leptoquarks involving lepton flavour violation at HERA may be found in $[3,5]$. 

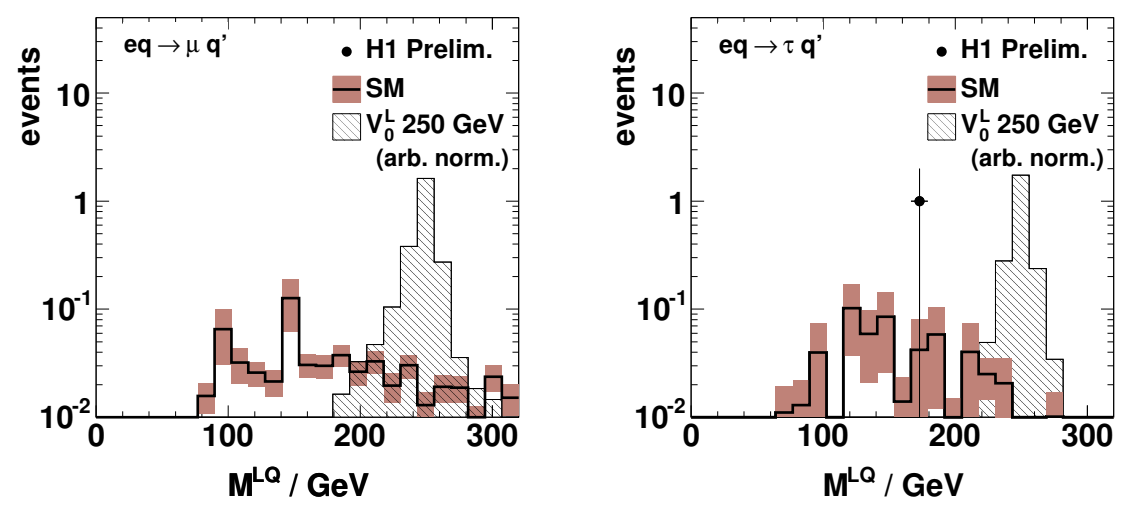

Figure 3: Muon-jet and tau-jet mass spectra.

(a)
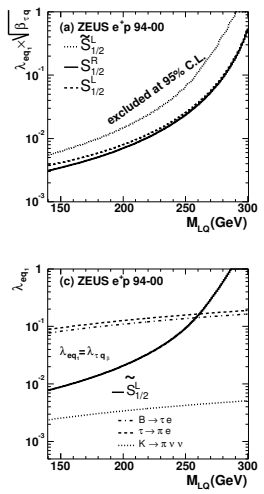

ZEUS
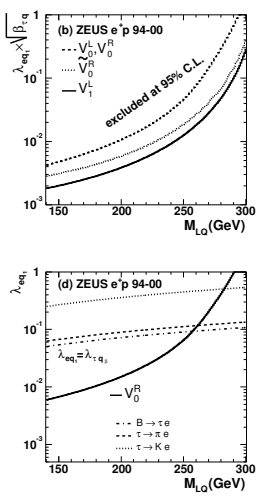

(b)

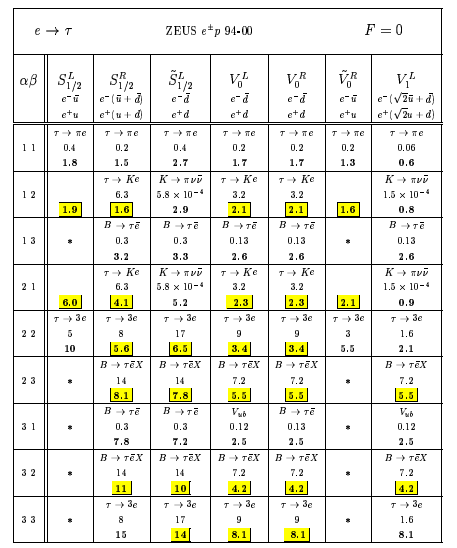

Figure 4: Limits on lepton-flavour violating leptoquarks involving $\tau$ leptons: (a) resonant case (b) contactinteraction limits (in $\mathrm{TeV}^{-2}$ ).

\section{Conclusions}

The data collected during the HERA I period (1994-2000) has been searched for leptoquark production in both lepton-flavour conserving and lepton-flavour violating decay modes. No signal has been found and a variety of models has been excluded, depending on the leptoquark mass and coupling.

\section{References}

[1] W. Buchmüller, R. Rückl, D. Wyler, Phys. Lett. B 191 (1987) 442 [Erratum-ibid, B 448 (1999) 320]

[2] S. Chekanov et al., Physical Review D 68 (2003)

[3] S. Chekanov et al., DESY-05-016, submitted to European Physical Journal

[4] A. Aktas et al., Phys. Lett. B 629 (2005) 9-19

[5] The H1 collaboration, Search for Lepton Flavor Violation in ep Collisions at HERA (conference paper), H1prelim-04-162 